\title{
Improving the screening and treatment of hypertension in people living with HIV: An evidence-based policy brief by Malawi's Knowledge Translation Platform
}

Collins Mitambo ${ }^{1,2}$, Shiraz Khan ${ }^{3}$, Beatrice L. Matanje-Mwagomba ${ }^{4}$, Chiyembekezo Kachimanga ${ }^{5}$, Emily Wroe ${ }^{5-7}$, Dalitso Segula ${ }^{8}$, Alemayehu Amberbir ${ }^{9,10}$, Daniela Garone ${ }^{10}$, Peter R.A. Malik ${ }^{10,11}$, Austrida Gondwe ${ }^{10}$, Josh Berman ${ }^{10}$

1. Directorate of Research, Ministry of Health, Lilongwe, Malawi

2. Public Health Institute of Malawi, Lilongwe, Malawi

3. UNC Project-Malawi, Lilongwe, Malawi

4. Directorate of Clinical Services, Ministry of Health, Lilongwe, Malawi

5. Abwenzi Pa Za Umoyo, Neno, Malawi

6. Partners in Health, Boston, Massachusetts, USA

7. Department of Medicine, Brigham and Women's Hospital, Boston, Massachusetts, USA

8. Malawi-Liverpool-Wellcome Trust Clinical Research Programme, Blantyre, Malawi

9. Malawi Epidemiology and Intervention Research Unit (MEIRU), Lilongwe, Malawi

10. Dignitas International, Zomba, Malawi

11. Faculty of Health Sciences and McMaster Health Forum, McMaster University, Hamilton, Ontario, Canada

Correspondence: Dr Collins Mitambo (cmitambo@gmail.com)

\section{Key messages}

\section{The problem:}

High burden of undiagnosed and untreated hypertension in people living with HIV in Malawi. There is high and increasing burden of hypertension in Malawi in the general population and specifically among people living with HIV. ${ }^{1,2}$ In Malawi, $32 \%$ of the adult population have been found to be hypertensive; $94 \%$ of these patients are unaware and therefore untreated. ${ }^{3}$ In a small HIV-infected cohort in Blantyre, $46 \%$ of patients were found to have elevated blood pressure. ${ }^{4}$ The lack of a well organised and funded national hypertension screening and treatment programme leaves hypertension in patients poorly managed.

\section{Policy options:}

Integration of hypertension management into the well-established HIV programme

The first three proposed options should be considered mutually exclusive, while the fourth is a complimentary option that can supplement the facility-based options outlined in options one through three:

1. Integration of hypertension management into well-established HIV programmes; this will involve hypertension screening and treatment by the same health workers at Antiretroviral Treatment (ART) clinics.

2. Hypertension screening within HIV clinics and subsequent referral to other chronic care management services within the same clinic or other facility.

3. Development of a comprehensive chronic care clinic model; this suggests a new health system arrangement where multiple Noncommunicable Diseases (NCDs) and HIV chronic care are combined and offered at one point of care delivered by the same health workers.
Included:

- Description of a health system problem

- Viable options for addressing this problem

- Strategies for implementing these options

Not included: recommendations

- This policy brief does not make recommendations regarding which policy option to choose

Who is this policy brief for?

Policymakers, their support staff, and other stakeholders with an interest in the problem addressed by this policy brief

Why was this policy brief prepared?

To inform deliberations about health policies and programmes by summarising the best available evidence about the problem and viable solutions

What is an evidence-based policy brief?

Evidence-based policy briefs bring together global research evidence (from systematic reviews* where possible) and local evidence to inform deliberations about health policies and programmes

*Systematic review: A summary of studies addressing a clearly formulated question that uses systematic and explicit methods to identify, select, and critically appraise the relevant research, and to collect and analyse data from this research

Executive Summary

The evidence presented in this executive summary is summarized from a full evidnece brief for policy.

4. The addition of hypertension screening and referral into community-based HIV activities; this option refers to community based activities aimed at capturing undiagnosed HIV infected and hypertensive patients and linking them to appropriate care.

The evidence for all four options is limited and the costs and cost-effectiveness of the four options are uncertain. Given the limitations of the currently available evidence, rigorous evaluation and monitoring of resource use and activities is warranted. 


\section{Implementation considerations:}

A combination of strategies is likely needed to effectively implement hypertension care within Malawi's HIV infected population

- Several key enablers should be taken into account: recognition of the NCD epidemic, well established national HIV services and existing HIV and NCD guidelines.

- Several barriers may jeopardize effective implementation of hypertension/HIV programs and will need to be actively addressed. This includes effects of HIV stigma, lack of health worker training, vertical HIV and NCD policies with limited collaboration, and finance as well as infrastructure development limitations. Strategies are suggested to mitigate these barriers within the full text.

\section{The problem}

There exists a high burden of undiagnosed and untreated hypertension within Malawi's HIV infected population. Africa is facing a growing double burden of communicable and noncommunicable diseases. It is projected that by 2025 nearly three-quarters of people with hypertension will be living in developing countries. ${ }^{1,5}$ Hypertension is expected to lead to more deaths in Africa than infectious diseases in the next 20-30 years. ${ }^{6,7}$ Malawi is facing a high prevalence of hypertension in the general population. ${ }^{3}$ Whilst only limited data exists, the burden of hypertension in Malawi in people living with HIV appears to be at least as common as in the general population. ${ }^{4}$

\section{Size of the problem}

In African adults, the rate of hypertension is reported to exceed $25 \%$ in many settings. According to the STEPs survey, around one third of Malawian adults were reported to have hypertension in 2009: often this was undiagnosed and therefore untreated. ${ }^{3}$ The burden of hypertension is likely to increase due to population transition effects, related to urbanization, increases in life expectancy, increasing wealth and associated dietary and life-style changes. ${ }^{2}$ Linkages between some NCDs and infectious diseases including HIV have also been noted. A small study from Blantyre estimated that $46 \%$ of people living with HIV had elevated blood pressure. $^{4}$

\section{Factors underlying the problem}

Several factors impact on the problem of hypertension in people living with HIV:

\section{Organization and delivery arrangement}

Malawi lacks a well-established national hypertension screening or treatment programme. Reliable diagnostic equipment and basic hypertensive medication are often not available in health centres. HIV and NCD programs are vertical with little or no integration.

\section{Patients and providers}

There is a limited training and mentorship available to health workers on the importance of screening for hypertension and its management. Malawi's HIV program has clinics that often are only treating HIV. The providers at these clinics are consistently overburdened. Hypertension diagnosis and treatment awareness among people living with HIV is also low.

\section{Financing arrangements}

Funding mechanisms may play an underlying role in affecting the problem as vertical HIV and ART clinics are well funded with no encouragement to manage hypertension.

http://dx.doi.org/10.4314/mmj.v29i2.27

\section{Four policy options}

As has been described elsewhere, ${ }^{8}$ multiple Communities of Practice were developed during the formation of KTPMalawi, including a committee to improve the screening and treatment of hypertension within HIV-infected populations. Each Community of Practice's mandate is to gather and synthesize the best available global and local evidence and produce evidence briefs for policy that have been used as the primary input into structured deliberative dialogues. The NCD-HIV Community of practice lays out below the best available local and global evidence on four policy options aimed at improving the active screening and management of hypertension among people living with HIV. The first three options described below are mutually exclusive, while the fourth can supplement the other health facility-based options.

\section{Policy option 1: Integration of hypertension screening and treatment into existing HIV clinic structures}

This policy option involves integrating hypertension screening and care into existing HIV services. All people living with HIV who are regularly seen by pre-ART or ART providers would be routinely screened and treated for hypertension.

HIV care has been proposed to provide a good opportunity for hypertension management.

From a health services perspective, chronic HIV infection and hypertension pose very similar challenges, as they both need long life treatment, regular monitoring and a reliable drug supply chain. Hypertension and HIV are also both diagnosed through simple screening tests and involve similar models of care.?

A search using multiple key words and databases as well as correspondence with experts in the field yielded no systematic reviews that discussed patient outcomes from the integration of hypertension and HIV screening and treatment. There is some evidence that specific NCDs may benefit from the strong systems already in place for HIV programs. ${ }^{10,11}$ A study from Ethiopia found that when HIVspecific standard operating protocols and provider support tools were adapted to NCD care, documentation improved dramatically. ${ }^{12}$ Several studies from Uganda, Tanzania, and Kenya that have found high levels of hypertension within HIV cohorts have argued for integrating screening and treatment within HIV clinics. ${ }^{13-15}$

Integration of care into one consultation may decrease costs to patients to access services, ${ }^{16}$ and may decrease health system costs by avoiding duplication but there is no strong evidence in support of this suggestion. There are likely to be upfront costs to integrating HIV and hypertension care and treatment which may include the development of support tools, training or re-training of the health workers, the provision of the requisite clinical equipment and consistent drug supplies. ${ }^{17}$

\section{Policy option 2: Hypertension screening within HIV clinics and subsequent referral}

People living with HIV could be screened for hypertension at pre-ART or ART clinics. Patients found to be hypertensive would then be referred to a general outpatient clinic or NCD clinic within the same health facility or other facilities if necessary for further management. Patients who are normotensive could be screened at regular intervals and Malawi Med J. 2017 Jun;29(2):224-228 
Table 1: Implementation considerations

\begin{tabular}{|c|c|}
\hline Barriers to implementation & Strategies for addressing implementation barriers \\
\hline $\begin{array}{l}\text { Limited financial resources to increase } \\
\text { screening and treatment of } \\
\text { hypertension and other NCD's }\end{array}$ & $\begin{array}{l}\text { - Support increased funding for NCD activities (26). } \\
\text { - Positioning chronic diseases in the development agenda } \\
\text { and increase mobilisation of resources } \\
\text { - Integrating hypertension care with well-funded vertical } \\
\text { programs (25). } \\
\text { - The already existing structures and investment in HIV } \\
\text { services in Malawi could potentially provide leverage for } \\
\text { integrating hypertension screening and management }\end{array}$ \\
\hline
\end{tabular}

Healthcare providers are not adequately trained and supervised to provide integrated hypertension-HIV services
- In-service training and mentorship for providers $(22,27)$.

- All HIV providers would need to receive in-service training and mentorship in the screening and management of hypertension

- Regular supervision, audits and feedback (12).

- Hypertension supervision and audits could be integrated into the existing well-developed HIV program audit and supervision program

\begin{tabular}{|l|l}
\hline Vertical HIV program design & Revision of existing management guidelines and policies (25). \\
& - Facilitate integrated care, HIV policies would need to be \\
& reviewed and updated to include management of other \\
& chronic conditions including hypertension
\end{tabular}

Loss to follow up

- All hypertensive patients should receive comprehensive health education and adherence counselling on NCDs (22).

- Introduce peer support programs and SMS reminders to support retention to care (28).

- Integrating HIV/NCD defaulters into the existing defaulter tracing programs (29).

Hypertensive patients may be hesitant to attend Chronic Care Clinic services due to HIV stigma
- The following approaches have been shown to reduce HIV stigma (30):

- Information-based approaches that concentrate on written information sharing and oral communication such as facility health talks, video etc.

- Skill-building approaches such as training with healthcare workers or community leaders, dramas etc.

- Contact strategies that link people living with HIV and the general public and

- Counselling/support for people living with HIV could receive health lifestyle advice. This option allows for identification of undiagnosed hypertension among people living with HIV with referral to the appropriate clinical care. There is limited documentation of programs integrating screening for hypertension and subsequent referral into HIV care models. ${ }^{13}$ In Neno District, Malawi, a pilot project has integrated hypertension screening into community and clinic based HIV activities. ${ }^{18,19}$ Early results show that this health system arrangement is feasible, however linkage to care is poor: $12 \%$ of community-screened patients were referred for hypertension management but only $20-25 \%$ of these referrals were enrolled in the NCD clinic.

Govindasamy et al. looked at screening for NCDs including hypertension during mobile HIV-testing campaigns in South Africa. ${ }^{20}$ The authors found $58.1 \%$ of the participants had elevated blood pressure and $50 \%$ of them linked to care.

There is need for more implementation research and piloting of this model of care to produce evidence of the possible advantages and disadvantages associated with screening and referral.

\section{Policy Option 3: Development of a comprehensive chronic care clinic model}

This policy option suggests a new model of care where multiple NCDs and HIV chronic care are merged into one chronic care clinic with all chronic conditions managed by the same healthcare worker.

In Malawi, there are some Chronic Care Clinic (CCC) models being piloted but only one site, in Neno, has included HIV in their CCC model. ${ }^{19}$ In Neno District, pre-existing ART clinics, which were functional at all 13 health facilities, were converted to a CCC model where patients with HIV and/or an NCD diagnosis (most commonly hypertension, asthma, epilepsy, diabetes, and heart failure) are seen for all of their conditions in one combined visit. Patients are also screened for comorbid conditions at the clinic. Implementation was completed in the first half of 2015 with early results expected late in the year.

The integrated NCD clinic at Kasungu District Hospital for instance, saw a $>100 \%$ rise in the size of the hypertension cohort and $>200 \%$ rise in the size of the diabetes patient 
cohorts between July 2012 and December 2013 (unpublished NCD program report, presented at $\mathrm{MOH}$ midyear review; April 2014). However, this NCD clinic has not integrated HIV services. ${ }^{21}$ In 2013 an integrated electronic medical record system for chronic NCDs including hypertension, diabetes, asthma and epilepsy, together with HIV was developed and is currently being piloted at two sites in Malawi.

A literature search found a few "lessons learned" papers, of which only one described a health system arrangement where HIV care has been integrated into a chronic care clinic model in Cambodia. ${ }^{22}$ The Cambodia CCC integrated care for HIV, hypertension and diabetes as well as epilepsy and thyroid disorders. Physicians and nurses received training in principles of chronic disease management and counsellors provided "a series of activities complementary to the medical consultations". There were "satisfactory outcomes" for all groups and importantly the authors noted that there was acceptability of the integrated model from both HIV and non-HIV patients.

There is need for more implementation research and piloting of this model of care to produce evidence of the possible advantages and disadvantages associated with chronic care clinics that incorporate HIV.

\section{Policy option 4: The addition of hypertension screening and referral into comunity-based HIV activities}

This policy option lays out a public health approach to capture undiagnosed HIV-infected and hypertensive patients and link them to appropriate health services. Both diseases can be screened for within community settings at low cost. In resource-limited settings, where health-seeking behavior is often poor and where there is limited access to quality chronic disease management, community based screening and referral to care may serve as an effective point of entry to care.

Although the Malawi HIV programme has several community-based activities that have been successful in improving HIV testing rates and supporting linkages to care, these national HIV community-screening activities have not been integrated with hypertension or any other disease screening effort. ${ }^{23}$

Community-based screening for multiple NCDs including hypertension and HIV has been shown to be feasible in one study in Kenya. ${ }^{24}$ Importantly, an informal survey of the counselors at the end of home-based screening revealed that participants who were initially reluctant to undergo HIV testing were more likely to be tested if they were offered screening for diabetes or hypertension at the same time. A study conducted in rural Uganda and Cape Town ${ }^{25}$ found that a community campaign with an integrated screening approach for multiple communicable and NCDs including hypertension, HIV, diabetes and malaria were feasible and highly accepted. However, this study showed that linkages to care after an elevated blood pressure diagnoses were suboptimal. This study showed that active case detection in the community can contribute to early hypertension treatment and care but promoting linkages to care must be continually addressed.

\section{Implementation considerations}

Employing any one of these policy options would necessitate significant changes in other areas of the health system that must be examined and planned for. Implementation strategies can capitalize on enablers of these policy options to improve the screening and treatment of hypertension among people living with HIV as well as addressing barriers to doing so.

Key enablers to improve the screening and treatment of hypertension among HIV infected patients include:

- Global recognition of the NCD epidemic in developing countries

- Emerging local evidence of the magnitude of the problem

- Well established HIV services at tertiary, district and primary-care levels

- Existing national guidelines for the management of chronic conditions including hypertension

- Inclusion of NCDs in Malawi's essential health package

- The rapid progress of Malawi NCD program and emerging NCD policies

Evidence regarding barriers to improving the screening and treatment of hypertension among people living with HIV and strategies to address these barriers are summarised in Table 1.

\section{Next Steps}

The aim of this policy brief is to foster dialogue and judgements that are informed by the best available evidence. The intention is not to advocate specific options or close off discussion. Further actions will flow from the deliberations that the policy brief is intended to inform. These might include:

- The need for further research on each of these policy options. Pilot programs could be developed in selected locations within Malawi and closely studied to elucidate patient outcomes, cost-effectiveness and unintended consequences.

- Development of clear guidance or policy on hypertension screening and treatment of HIV infected patients.

\section{Acknowledgements}

This policy brief was prepared with the aid of a grant from the International Development Research Centre, Ottawa, Canada, the Health Research Capacity Strengthening Initiative (HRCSI) hosted by the Malawi National Commission for Science and Technology, Grand Challenges Canada and USAID-Malawi.We would like to thank the following people for providing us with input and feedback:

Dr Joep van Oosterhout, Caitlyn Timmings, Dr Julia Moore, Prof. Shabbar Jaffar, Dr Mia Crampin, Dr Damson Kathyola, Dr Lonia Mwape, Dr Martha Masamba, Dr Lisa Puchalski Ritchie, KTPMalawi Steering Committee members

\section{Competing interests}

None of the authors reported a conflict of interest

This policy brief was prepared by Malawi's Knowledge Translation Platform's Noncommunicable disease - HIV Community of Practice, under the EVIPNet programme.

The Evidence-Informed Policy Network (EVIPNet) promotes the use of health research in policymaking. Focusing on low- and middle-income countries, EVIPNet promotes partnerships at the country level between policymakers, researchers and civil society in order to facilitate policy development and implementation through the use of the best scientific evidence available. http://www.who.int/evidence/about/en/ 


\section{References}

1. Kearney PM, Whelton M, Reynolds K, Muntner P, Whelton PK, He J. Global Burden of Hypertension: Analysis of Worldwide Data. The Lancet. 2005 Jan 15;365(9455):217-23

2. Matchaya GC. Trends in life expectancy and the macroeconomy in Malawi MPRA. Paper No. 11949, posted 27 December 2008. Retrieved from: http://mpra.ub.uni-muenchen.de/11949/.

3. Msyamboza KP, Ngwira B, Dzowela T, Mvula C, Kathyola D, Harries $\mathrm{AD}$, Bowie $\mathrm{C}$. The burden of selected chronic noncommunicable diseases and their risk factors in Malawi: nationwide STEPS survey. PLoS One. 2011 May 23;6(5):e20316.

4. Muronya W, Sanga E, Talama G, Kumwenda JJ, van Oosterhout JJ. Cardiovascular risk factors in adult Malawians on long-term antiretroviral therapy. Transactions of the Royal Society of Tropical Medicine and Hygiene. 2011 Nov 1;105(11):644-9.

5. Lloyd-Sherlock P, Beard J, Minicuci N, Ebrahim S, Chatterji S. Hypertension among older adults in low-and middle-income countries: prevalence, awareness and control. International journal of epidemiology. 2014 Feb 1;43(1):116-28.

6. Ataklte F, Erqou S, Kaptoge S, Taye B, Echouffo-Tcheugui JB, Kengne AP. Burden of undiagnosed hypertension in Sub-Saharan Africa A systematic review and meta-analysis. Hypertension. 2015 Feb 1;65(2):291-8.

7. World Health Organization (WHO). Countries - Malawi (2012). Retrieved from http://www.who.int/countries/mwi/en/. 2012.

8. Berman J, Mitambo C, Matanje-Mwagomba B, Khan S, Kachimanga $\mathrm{C}$, Wroe $\mathrm{E}$, et al. Building a knowledge translation platform in Malawi to support evidence-informed health policy. Health Research Policy and Systems. 2015 Dec 8;13(1):1.

9. Haregu TN, Setswe G, Elliott J, Oldenburg B. National Responses to HIV/AIDS and Noncommunicable Diseases in Developing Countries: Analysis of Strategic Parallels and Differences. Journal of Public Health Research. 2014 Mar 26;3(1).

10. Oti SO. HIV and noncommunicable diseases: a case for health system building. Current Opinion in HIV and AIDS. 2013 Jan 1;8(1):659.

11. Remais JV, Zeng G, Li G, Tian L, Engelgau MM. Convergence of noncommunicable and infectious diseases in low-and middle-income countries. International Journal of Epidemiology. 2012 Oct 13:dys135.

12. Rabkin M, Melaku Z, Bruce K, Reja A, Koler A, Tadesse Y, Kamiru HN, Sibanyoni LT, El-Sadr W. Strengthening Health Systems for Chronic Care: Leveraging HIV Programs to Support Diabetes Services in Ethiopia and Swaziland. Journal of Tropical Medicine. 2012 Sep 27;2012.

13. Harries AD, Zachariah R, Jahn A, Schouten EJ, Kamoto K. Scaling up Antiretroviral Therapy in Malawi-Implications for Managing other Chronic Diseases in Resource-Limited Countries. Journal of Acquired Immune Deficiency Syndromes. 2009 Nov 1;52:S14-6.

14. Peck RN, Shedafa R, Kalluvya S, Downs JA, Todd J, Suthanthiran M, Fitzgerald DW, Kataraihya JB. Hypertension, kidney disease, HIV and antiretroviral therapy among Tanzanian adults: a cross-sectional study. BMC medicine. 2014 Jul 29;12(1):1.

15. Mateen FJ, Kanters S, Kalyesubula R, Mukasa B, Kawuma E, Kengne AP, Mills EJ. Hypertension prevalence and Framingham risk score stratification in a large HIV-positive cohort in Uganda. Journal of hypertension. $2013 \mathrm{Jul}$ 1;31(7):1372-8.
16. Hyle EP, Naidoo K, Su AE, El-Sadr WM, Freedberg KA. HIV, Tuberculosis, and Noncommunicable Diseases: What is known about the costs, effects, and cost-effectiveness of integrated care?. Journal of acquired immune deficiency syndromes (1999). 2014 Sep 1;67(0 1):S87.

17. Lamptey P, Dirks R. Building on the AIDS response to tackle noncommunicable disease. Global Heart. 2012 Mar 1;7(1):67-71.

18. PIH. Partners in Health Data from Neno, Malawi, September 2014. Unpublished Monitoring Data.

19. Wroe E., Kachimanga, C., Mailosi, B., Gonani, G., et al. Novel strategies for improving case finding and access to care for HIV-positive patients with noncommunicable diseases in rural Malawi. AIDS conference. 2014

20. Govindasamy D, Kranzer K, van Schaik N, Noubary F, Wood R, Walensky RP, Freedberg KA, Bassett IV, Bekker LG. Linkage to HIV, TB and noncommunicable disease care from a mobile testing unit in Cape Town, South Africa. PLoS One. 2013 Nov 13;8(11):e80017.

21. MOH Malawi. NCD programme mid year review, Malawi. 2014.

22. Janssens B, Van Damme W, Raleigh B, Gupta J, Khem S, Soy Ty K, Vun MC, Ford N, Zachariah R. Offering integrated care for HIV/AIDS, diabetes and hypertension within chronic disease clinics in Cambodia. Bulletin of the World Health Organization. 2007 Nov; $85(11): 880-5$

23. Malawi Government. Global AIDS response progress report - Malawi country report for 2010-2011.

24. Pastakia SD, Ali SM, Kamano JH, Akwanalo CO, Ndege SK, Buckwalter VL, Vedanthan R, Bloomfield GS. Screening for diabetes and hypertension in a rural low income setting in western Kenya utilizing home-based and community-based strategies. Globalization and health. 2013 May 16;9(1):1.

25. Chamie G, Kwarisiima D, Clark TD, Kabami J, Jain V, Geng E, Petersen ML, Thirumurthy H, Kamya MR, Havlir DV, Charlebois ED. Leveraging rapid community-based HIV testing campaigns for noncommunicable diseases in rural Uganda. PloS one. 2012 Aug 20;7(8):e43400.

26. Geneau R, Stuckler D, Stachenko S, McKee M, Ebrahim S, Basu S, Chockalingham A, Mwatsama M, Jamal R, Alwan A, Beaglehole R. Raising the priority of preventing chronic diseases: a political process. The Lancet. 2010 Nov 19;376(9753):1689-98.

27. Bluestone J, Johnson P, Fullerton J, Carr C, Alderman J, BonTempo J. Effective in-service training design and delivery: evidence from an integrative literature review. Human resources for health. 2013 Oct $1 ; 11(1): 1$.

28. Horvath T, Azman H, Kennedy GE, Rutherford GW. Mobile phone text messaging for promoting adherence to antiretroviral therapy in patients with HIV infection. The Cochrane Library. 2012 Jan 1.

29. Thomson KA, Cheti EO, Reid T. Implementation and outcomes of an active defaulter tracing system for HIV, prevention of mother to child transmission of HIV (PMTCT), and TB patients in Kibera, Nairobi, Kenya. Transactions of the Royal Society of Tropical Medicine and Hygiene. 2011 Jun 1;105(6):320-6.

30. Brown L, Macintyre K, Trujillo L. Interventions to reduce HIV/ AIDS stigma: what have we learned? AIDS education and prevention. 2003 Feb 1;15(1):49-69. 